\title{
Paroxysmal Sympathetic Hyperactivity: An Old but Unrecognized Condition
}

\author{
Filipa Brás Monteiro, Ricardo Castro Fonseca, Rita Mendes
}

Internal Medicine Department, Hospital Egas Moniz, Centro Hospitalar Lisboa Ocidental, Lisbon, Portugal

Received: 29/12/2016

Accepted: 04/01/2017

Published: 30/01/2017

How to cite this article: Monteiro F, Fonseca R, Mendes R. Paroxysmal sympathetic hyperactivity: an old but unrecognized condition. EJCRIM 2017;4: doi:10.12890/2017_000562

Conflicts of Interests: The Authors declare that there are no competing interests.

This article is licensed under a Commons Attribution Non-Commercial 4.0 License

\section{ABSTRACT}

Patients who survive a traumatic brain injury (TBI) can sometimes experience symptoms of excessive sympathetic discharge. Despite being known about for more than 60 years, the diagnostic criteria for this condition have only recently been defined under the name "paroxysmal sympathetic hyperactivity". Failure to recognize this syndrome leads to excessive costs, prolonged hospital stays and delayed recovery for TBI patients. This case report describes a patient whose specific rehabilitation program was affected by a failure to identify this entity, even though he presented with many of the characteristics of this condition.

\section{LEARNING POINTS}

- Fever is a common sign in hospitalized patients and can result in expensive investigations and prolong length of stay.

- It is crucial to consider paroxysmal sympathetic hyperactivity (PSH) as a cause of fever in traumatic brain injury patients (TBI).

- A delay in diagnosing and treating PSH can have devastating consequences for these patients' recovery programs.

\section{KEYWORDS}

Paroxysmal sympathetic hyperactivity (PSH), traumatic brain injury (TBI), fever, beta-blocker

\section{CASE PRESENTATION}

The authors report the case of 27-year-old male who was aphasic and tetraplegic following a traumatic brain injury (TBI) with severe right hemisphere lesions sustained in a road traffic collision. Five months after the accident, the patient was readmitted to hospital because of nosocomial pneumonia and was started on a 10-day course of the antibiotic meropenem. No pathogens were isolated from blood or sputum cultures and the patient improved clinically to be stable on air, with no respiratory symptoms and negative inflammatory markers.

Despite the apparently treated infection, the patient was still febrile with his temperature rising to $39.5^{\circ} \mathrm{C}$ once or twice a day with very little response to antipyretic drugs. During these episodes, the patient became tachycardic, hypertensive, tachypnoeic and sweated profusely. The patient's mother reported that these episodes were also frequent at home and that because of them, the patient had been refused care in a specialized rehabilitation hospital as he was thought to be infectious.

A complete septic screen was performed including blood, sputum (obtained from bronchoscopy), urine and liquor samples but no bacteria, yeast or fungus were found, and no changes were seen on imaging tests. The inflammatory markers were also negative: white cell count $7,000 \times 10^{9} /$ (normal value $<11,000 \times 109 /$ ) , C-reactive protein $<0.5 \mathrm{mg} / \mathrm{dl}$ (normal value $<0.5 \mathrm{mg} / \mathrm{dl}$ ) and erythrocyte sedimentation rate 23 $\mathrm{mm} / \mathrm{h}$ (normal value $<20 \mathrm{~mm} / \mathrm{h}$ ). Both renal and liver function were normal.

Paroxysmal sympathetic hyperactivity (PSH) was then considered as the cause of the patient's febrile episodes. PSH is a common but unrecognized complication after TBI and is characterized by hypertension, tachycardia, sweating, tachypnoea and hyperthermia, the last 
being less frequent than the other signs ${ }^{[1]}$.

A consensus statement was issued only recently on the definition, nomenclature and diagnostic criteria for PSH, an entity that was previously poorly recognized, thus leading to delays in treatment and increased morbidity among TBI patients. In this consensus, an assessment measure was proposed combining a "Clinical Feature Scale" and a "Diagnosis Likelihood Tool"[2]. Our patient scored 22 points in this proposed model, indicating a 'Probable' PSH diagnosis (Table 1).

Current state-of-the art treatment for this condition with beta-blockers was initiated with propranolol 10 mg every 8 hours up-titrated to $30 \mathrm{mg}$ every 6 hours on discharge ${ }^{[3]}$. The patient experienced complete resolution of all the described symptoms and no negative betablocker side-effects were noticed.

\begin{tabular}{|c|c|c|c|c|c|}
\hline \multicolumn{6}{|l|}{ Clinical Feature Scale (CSF) } \\
\hline & 0 & 1 & 2 & 3 & Score \\
\hline Heart Rate & $<100$ & $100-119$ & $120-139$ & $\geq 140$ & 2 \\
\hline Respiratory rate & $<18$ & $18-23$ & $24-29$ & $\geq 30$ & 2 \\
\hline Systolic blood pressure & $<140$ & $140-159$ & $160-179$ & $\geq 180$ & 3 \\
\hline Temperature & $<37$ & $37-37.9$ & $38-38.9$ & $\geq 39.0$ & 2 \\
\hline Sweating & $\mathrm{Na}$ & Mild & Moderate & Severe & 3 \\
\hline Posturing during episodes & $\mathrm{Na}$ & Mild & Moderate & Severe & 1 \\
\hline & & & \multicolumn{2}{|c|}{ CSF subtotal } & 13 \\
\hline \multirow{4}{*}{ Severity of clinical features } & & & Nil & 0 & \\
\hline & & & Mild & $0-6$ & \\
\hline & & & Moderate & $7-12$ & \\
\hline & & & Severe & $\geq 13$ & $x$ \\
\hline
\end{tabular}

Diagnosis Likelihood Tool (DLT)

Clinical features occur simultaneously

Episodes are paroxysmal in nature

Sympathetic over-reactivity to normally non-painful stimuli

Features persist $\geq 3$ consecutive days

Features persist $\geq 2$ weeks post brain injury

Features persist despite treatment of alternative differential diagnosis

Medication administered to decrease sympathetic features

$\geq 2$ episodes daily

Absence of parasympathetic features during episodes

Absence of other presumed cause of features

Antecedent acquired brain injury

\begin{tabular}{l|l} 
(Score 1 point for each feature present) & DLT subtotal
\end{tabular}

\begin{tabular}{|c|c|}
\hline Combined total (CSF+DLT) & 22 \\
\hline
\end{tabular}

\begin{tabular}{|c|c|c|c|}
\hline \multirow{3}{*}{ PSH diagnostic likelihood } & Unlikely & $<8$ & \\
\hline & Possible & $8-16$ & \\
\hline & Probable & $>17$ & $x$ \\
\hline
\end{tabular}

Table 1. Paroxysmal sympathetic hyperactivity assessment measure

PSH, Paroxysmal sympathetic hyperactivity

\section{DISCUSSION}

PSH has only been defined recently ${ }^{[2]}$. Previously, the lack of a definition and diagnostic criteria resulted in a poorly understood condition with a variety of symptoms that made recognition difficult. Although it has been known about for more than 60 years, it had over 31 different eponyms, making it very difficult to identify ${ }^{[2,4]}$.

Recognition of this syndrome is crucial for faster recovery in TBI patients, even while still in intensive care units, thus reducing complication rates and length of stay ${ }^{[5]}$.

This is a common syndrome and failure to recognize it is associated with increased morbidity and mortality, higher health costs, longer hospitalizations and poorer outcomes ${ }^{[2]}$. This case report highlights the implications of missing this diagnosis as the patient had previously been denied admittance to a rehabilitation hospital. 


\section{REFERENCES}

1. Mathew MJ, Deepika A, Shukla D, Devi BI, Ramesh VJ. Paroxysmal sympathetic hyperactivity in severe traumatic brain injury. Acta Neurochir $2016 ; 158: 2047-2052$.

2. Baguley IJ, Perkes IE, Fernandez-Ortega J-F, Rabinstein AA, Dolce G, Hendricks HT, for the Consensus Working Group. Paroxysmal sympathetic hyperactivity after acquired brain injury: consensus on conceptual definition, nomenclature, and diagnostic criteria. J Neurotrauma 2014;31:1515-1520.

3. Samuel S, Allison TA, Lee K, Choi HA. Pharmacological management of paroxysmal sympathetic hyperactivity. J Neurosci Nurs 2016;48:82-89.

4. Perkes I, Baguley IJ, Nott MT, Menon DK. A review of paroxysmal sympathetic hyperactivity after acquired brain injury. Ann Neurol $2010 ; 68: 126-135$.

5. Meyer KS. Understanding paroxysmal sympathetic hyperactivity after traumatic brain injury. Surg Neurol Int 2014;5(Suppl 13):S490-492. 1 